Yi-Ru Fu, Jian Luan and Xiao-Jie Wang*

\title{
The crystal structure of tris(1-ethylimidazole- $\kappa^{1} N$ )- (sulfato- $\mathrm{K}^{2} \mathrm{O}, \mathrm{O}^{\prime}$ ) vanadium(IV), $\mathrm{C}_{15} \mathrm{H}_{24} \mathrm{~N}_{6} \mathrm{O}_{5} \mathrm{SV}$
}

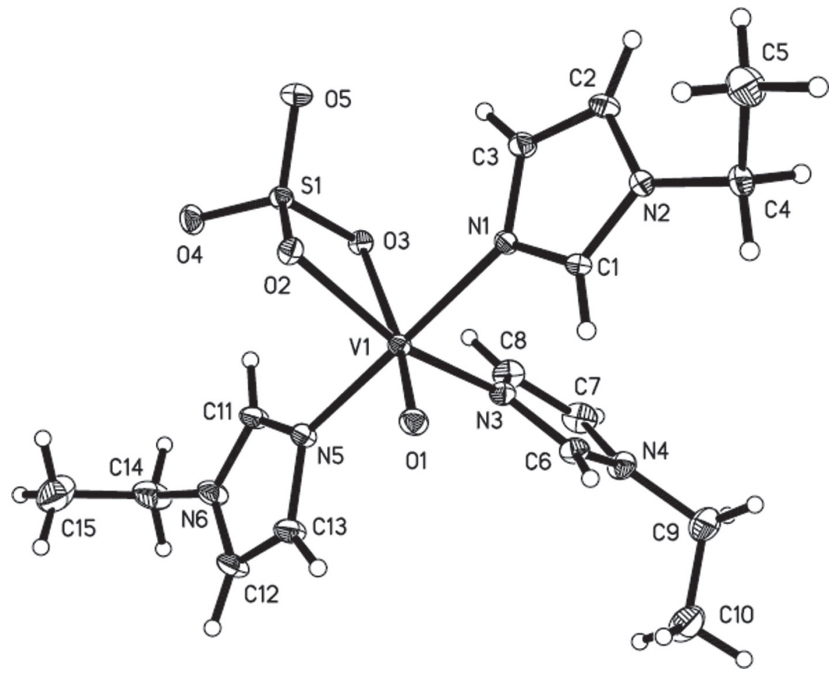

https://doi.org/10.1515/ncrs-2020-0095

Received February 17, 2020; accepted March 10, 2020; available online April 9, 2020

\section{Abstract}

$\mathrm{C}_{15} \mathrm{H}_{24} \mathrm{~N}_{6} \mathrm{O}_{5} \mathrm{SV}$, monoclinic, $P 2_{1} / c$ (no. 14), $a=16.0059 \AA$, $b=9.1008 \AA, c=14.2686 \AA$ 影 $\beta=100.352^{\circ}, V=2044.6(2) \AA^{3}$, $Z=4, R_{\text {gt }}(F)=0.0400, w R_{\text {ref }}\left(F^{2}\right)=0.0852, T=173(2) \mathrm{K}$.

\section{CCDC no.: 1989326}

The complex title structure is shown in the figure. Table 1 contains crystallographic data and Table 2 contains the list of the atoms including atomic coordinates and displacement parameters.

\section{Source of material}

$\mathrm{VOSO}_{4} \cdot 2 \mathrm{H}_{2} \mathrm{O}(0.199 \mathrm{~g}, 1 \mathrm{mmol})$ in 1-ethylimidazole $(6 \mathrm{~mL})$ was sealed in a $20 \mathrm{~mL}$ vial, which was heated to $90^{\circ}$ under autogenously pressure for $72 \mathrm{~h}$. Yield: $60 \%$ based on V. Anal.

\footnotetext{
*Corresponding author: Xiao-Jie Wang, College of Chemistry, Chemical Engineering and Environmental Engineering, Liaoning Shihua University, Fushun, Liaoning Province 113001, P.R. China, e-mail: wangxiaojie@lnpu.edu.cn. https://orcid.org/0000-00020405-8563

Yi-Ru Fu and Jian Luan: College of Chemistry, Chemical Engineering and Environmental Engineering, Liaoning Shihua University, Fushun, Liaoning Province 113001, P.R. China
}

Table 1: Data collection and handling.

\begin{tabular}{ll}
\hline Crystal: & Blue plate \\
Size: & $0.19 \times 0.16 \times 0.07 \mathrm{~mm}$ \\
Wavelength: & Mo $K \alpha$ radiation $(0.71073 \AA)$ \\
$\mu:$ & $0.63 \mathrm{~mm}^{-1}$ \\
Diffractometer, scan mode: & $\mathrm{CCD}, \varphi$ and $\omega$ \\
$\theta_{\text {max }}$, completeness: & $25.3^{\circ}, 99 \%$ \\
$N\left(h k l_{\text {measured }}, N(h k l)_{\text {unique }}, R_{\text {int }}:\right.$ & $11434,3692,0.063$ \\
Criterion for $I_{\text {obs }}, N(h k l)_{\text {gt }}:$ & $I_{\text {obs }}>2 \sigma\left(I_{\text {obs }}\right), 2699$ \\
$N(\text { param })_{\text {refined }}:$ & 256 \\
Programs: & Bruker [1], SHELX [2], Olex2 [3] \\
\hline
\end{tabular}

Table 2: Fractional atomic coordinates and isotropic or equivalent isotropic displacement parameters $\left(\AA^{2}\right)$.

\begin{tabular}{lrrrr}
\hline Atom & $\boldsymbol{x}$ & $\boldsymbol{y}$ & $\boldsymbol{z}$ & \multicolumn{1}{c}{$\boldsymbol{U}_{\text {iso }} / \boldsymbol{U}_{\text {eq }}$} \\
\hline C1 & $0.64695(19)$ & $0.3047(3)$ & $0.3291(2)$ & $0.0198(7)$ \\
H1 & 0.688429 & 0.275393 & 0.292903 & $0.024^{*}$ \\
C2 & $0.5264(2)$ & $0.3790(3)$ & $0.3655(2)$ & $0.0257(7)$ \\
H2 & 0.469309 & 0.410915 & 0.361046 & $0.031^{*}$ \\
C3 & $0.58492(19)$ & $0.3600(3)$ & $0.4466(2)$ & $0.0235(7)$ \\
H3 & 0.575105 & 0.376268 & 0.509541 & $0.028^{*}$ \\
C4 & $0.5290(2)$ & $0.3381(4)$ & $0.1892(2)$ & $0.0293(8)$ \\
H4A & 0.484774 & 0.260872 & 0.178396 & $0.035^{*}$ \\
H4B & 0.573489 & 0.311682 & 0.152089 & $0.035^{*}$ \\
C5 & $0.4900(2)$ & $0.4836(4)$ & $0.1540(2)$ & $0.0452(10)$ \\
H5A & 0.444471 & 0.508290 & 0.188907 & $0.068^{*}$ \\
H5B & 0.466638 & 0.476425 & 0.085822 & $0.068^{*}$ \\
H5C & 0.533645 & 0.560311 & 0.164514 & $0.068^{*}$ \\
C6 & $0.74230(19)$ & $-0.0441(3)$ & $0.4228(2)$ & $0.0243(7)$ \\
H6 & 0.759494 & -0.012938 & 0.365597 & $0.029^{*}$ \\
C7 & $0.6967(2)$ & $-0.1823(4)$ & $0.5275(2)$ & $0.0333(8)$ \\
H7 & 0.676364 & -0.264023 & 0.558284 & $0.040^{*}$ \\
C8 & $0.7129(2)$ & $-0.0469(4)$ & $0.5641(2)$ & $0.0283(8)$ \\
H8 & 0.705379 & -0.016325 & 0.625675 & $0.034^{*}$ \\
C9 & $0.7073(2)$ & $-0.3020(4)$ & $0.3686(3)$ & $0.0440(10)$ \\
H9A & 0.678455 & -0.385569 & 0.393776 & $0.053^{*}$ \\
H9B & 0.671101 & -0.269782 & 0.308367 & $0.053^{*}$ \\
C10 & $0.7896(3)$ & $-0.3522(4)$ & $0.3482(3)$ & $0.0508(11)$ \\
H10A & 0.817870 & -0.270703 & 0.321685 & $0.076^{*}$ \\
H10B & 0.780307 & -0.433001 & 0.302055 & $0.076^{*}$ \\
H10C & 0.825276 & -0.386443 & 0.407219 & $0.076^{*}$ \\
C11 & $0.8997(2)$ & $0.1735(4)$ & $0.7014(2)$ & $0.0262(7)$ \\
H11 & 0.859245 & 0.198646 & 0.740012 & $0.031^{*}$ \\
C12 & $1.0167(2)$ & $0.0960(4)$ & $0.6582(2)$ & $0.0309(8)$ \\
H12 & 1.072075 & 0.057659 & 0.659553 & $0.037^{*}$ \\
C13 & $0.9616(2)$ & $0.1415(4)$ & $0.5806(2)$ & $0.0286(8)$ \\
H13 & 0.972064 & 0.140214 & 0.517154 & $0.034^{*}$ \\
C14 & $1.0140(2)$ & $0.0929(5)$ & $0.8359(2)$ & $0.0440(10)$ \\
H14A & 1.046147 & -0.000599 & 0.843043 & $0.053^{*}$
\end{tabular}


Table 2 (continued)

\begin{tabular}{lrrrr}
\hline Atom & $\boldsymbol{x}$ & $\boldsymbol{y}$ & $\boldsymbol{z}$ & $\boldsymbol{U}_{\text {iso }} \boldsymbol{U}_{\text {eq }}$ \\
\hline H14B & 0.967887 & 0.085482 & 0.873534 & $0.053^{*}$ \\
C15 & $1.0721(3)$ & $0.2170(5)$ & $0.8733(3)$ & $0.0596(13)$ \\
H15A & 1.118376 & 0.223056 & 0.836861 & $0.089^{*}$ \\
H15B & 1.095709 & 0.199754 & 0.940610 & $0.089^{*}$ \\
H15C & 1.040119 & 0.309428 & 0.866771 & $0.089^{*}$ \\
N1 & $0.66062(15)$ & $0.3136(3)$ & $0.42325(16)$ & $0.0175(5)$ \\
N2 & $0.56687(16)$ & $0.3426(3)$ & $0.29145(17)$ & $0.0216(6)$ \\
N3 & $0.74200(15)$ & $0.0407(3)$ & $0.49793(17)$ & $0.0206(6)$ \\
N4 & $0.71516(18)$ & $-0.1797(3)$ & $0.43794(19)$ & $0.0285(7)$ \\
N5 & $0.88789(16)$ & $0.1901(3)$ & $0.60755(17)$ & $0.0211(6)$ \\
N6 & $0.97685(17)$ & $0.1161(3)$ & $0.73432(18)$ & $0.0293(7)$ \\
01 & $0.83011(13)$ & $0.2731(2)$ & $0.42317(14)$ & $0.0237(5)$ \\
02 & $0.78286(13)$ & $0.4676(2)$ & $0.56484(13)$ & $0.0204(5)$ \\
03 & $0.71898(12)$ & $0.2765(2)$ & $0.63494(13)$ & $0.0191(5)$ \\
04 & $0.80374(13)$ & $0.4561(2)$ & $0.73786(14)$ & $0.0274(5)$ \\
05 & $0.66665(13)$ & $0.5260(2)$ & $0.64964(15)$ & $0.0271(5)$ \\
S1 & $0.74209(5)$ & $0.43673(8)$ & $0.65095(5)$ & $0.01873(18)$ \\
V1 & $0.77923(3)$ & $0.26022(6)$ & $0.50944(3)$ & $0.01657(13)$ \\
\hline
\end{tabular}

Calcd. for $\mathrm{C}_{15} \mathrm{H}_{24} \mathrm{~N}_{6} \mathrm{O}_{5} \mathrm{SV}$ (\%): C, 39.91; H, 5.36; N, 18.62 . Found: C, 39.78; H, 5.51; N, 18.49.

\section{Experimental details}

The structure was solved by direct methods with the SHELXS program. All H-atoms were positioned with idealized geometry and refined isotropic $\left(U_{\text {iso }}(\mathrm{H})=1.2 U_{\text {eq }}(\mathrm{C})\right)$, using a riding model with $\mathrm{C}($ imodazole $)-\mathrm{H}=0.950 \AA$, $\mathrm{C}($ methylene $)-$ $\mathrm{H}=0.99 \AA$ and $\mathrm{C}($ methyl $)-\mathrm{H}=0.98 \AA$, respectively.

\section{Comment}

The range of potential applications of vanadium complexes range from catalysis to bioactivity [4-10]. The design and synthesis of vanadium complexes will be very meaningful. In this text, we describe the synthesis of an interesting vanadium complex by a simple solvothermal reaction.

The central vanadium atom adopts a ' $4+1+1$ ' geometry formed by three $\mathrm{N}$ atoms from1-eIM, two bridging oxygen $\mathrm{O}_{\mathrm{b}}$ from sulfate and one terminal oxygen $\mathrm{O}_{\mathrm{t}}$ with the $\mathrm{V}-\mathrm{O}$ and $\mathrm{V}-$ $\mathrm{N}$ bonds within the range of 1.597-2.186 Å and 2.082-2.125 $\AA$, respectively [11]. The sulfate ligand is coordinated in a chelating bidentate mode with the $\mathrm{S}-\mathrm{O}$ bonds within $1.448-1.518 \AA$. Although the central vanadium possesses distorted octahedral coordination geometry which is same to the previous reports in the similar system [12]. But in the previous reports, the four coordinated equatorial atoms are $\mathrm{N}$ atoms from 1-eIM. It is noteworthy that the 1-eIM plays a dual function as solvent and ligands.
Acknowledgements: This work was supported by the National Natural Science Foundation of China (no. 21401111) and the Natural Science Foundation of Liaoning Shihua University (2019XJJL-019).

\section{References}

1. Bruker. SAINTv8.37A. Bruker AXS Inc, Madison, WI, USA (2015).

2. Sheldrick, G. M.: Crystal structure refinement with SHELXL. Acta Crystallogr. C71 (2015) 3-8.

3. Dolomanov, O. V.; Bourhis, L. J.; Gildea, R. J.; Howard, J. A. K.; Puschmann, H.: OLEX2: a complete structure solution, refinement and analysis program. J. Appl. Crysallogr. 42 (2009) 339-341.

4. Barthelet, K.; Riou, D.; Ferey, G.: $\left[\mathrm{V}^{\prime \prime \prime}\left(\mathrm{H}_{2} \mathrm{O}\right)\right]_{3} \mathrm{O}\left(\mathrm{O}_{2} \mathrm{CC}_{6} \mathrm{H}_{4} \mathrm{CO}_{2}\right)_{3} \cdot\left(\mathrm{Cl}, 9 \mathrm{H}_{2} \mathrm{O}\right)(\mathrm{MIL}-59)$ : a rare example of vanadocarboxylate with a magnetically frustrated three-dimensional hybrid framework. Chem. Commun. 14 (2002) 1492-1493.

5. Laye, R. H.; Wei, Q.; Mason, P. V.; Shanmugam, M.; Teat, S. J.; Brechin, E. K.; Collison, D.; McInnes, E. J. L.: A highly reduced vanadium(III/IV) polyoxovanadate comprising an octavanadyl square-prism surrounding a dimetallic vanadium(III) fragment. J. Am. Chem. Soc. 128 (2006) 9020-9021.

6. Hu, X. K.; Wang, H.; Huang, B.; Li, N.; Hu, K. H.; Wu, B. L.; Xiao, Z. C.; Wei, Y. H.; Wu, P. F.: A new scheme for rational design and synthesis of polyoxovanadate hybrids with high antitumor activities. J. Inorg. Biochem. 193 (2019) 130-132.

7. Steens, N.; Ramadan, A. M.; Absillis, G.; Parac-Vogt, T. N.: Hydrolytic cleavage of DNA-model substrates promoted by polyoxovanadates. Dalton Trans. 39 (2010) 585-592.

8. Boulmier, A.; Feng, X. X.; Oms, O.; Mialane, P.; Riviere, E.; Shin, C. J.; Yao, J. Q.; Kubo, T.; Furuta, T.; Oldfield, E.; Dolbecq, A.: Anticancer activity of polyoxometalate-bisphosphonate complexes: synthesis, characterization, in vitro and in vivo results. Inorg. Chem. 56 (2017) 7558-7565.

9. Dankhoff, K.; Ahmad, A.; Weber, B.; Biersack, B.; Schobert, R.: Anticancer properties of a new non-oxido vanadium(IV) complex with a catechol-modified 3,3'-diindolylmethane ligand. . . Inorg. Biochem. 194 (2019) 1-6.

10. Wang, L. J.; Fan, H. T.; Du, C. J.; Xing, X. J.; Zhao, Y. Y.; Chen, B. K.; Wang, L. Y.: Synthesis, structure and properties of a co-crystallized complex based on polyoxovanadate $\left[\mathrm{V}^{\mathrm{IV}}{ }_{12} \mathrm{~V}_{6} \mathrm{O}_{42}\right]^{6-}$ and a mononuclear vanadium complex [VON $\left(\mathrm{CH}_{2} \mathrm{CH}_{2} \mathrm{O}\right)_{3}$ ]. Inorg. Chem. Commun. 63 (2016) 39-41.

11. Biswal, D.; Pramanik, N. R.; Chakrabarti, S.; Drew, M. G. B.; Acharya, K.; Chandra, S.: Syntheses, crystal structures, DFT calculations, protein interaction and anticancer activities of water soluble dipicolinic acid-imidazole based oxidovanadium(iv) complexes. Dalton Trans. 46 (2017) 16682-16702.

12. Chen, B. K.; Huang, X. Q.; Wang, B.; Lin, Z. G.; Hu, J. F.; Chi, Y. N.; Hu, C. W.: Three new imidazole-functionalized hexanuclear oxidovanadium clusters with exceptional catalytic oxidation properties for alcohols. Chem. Eur. J. 19 (2013) 4408-4413. 